\title{
Confinement of Screw Dislocations to Predetermined Lateral Positions in (0001) 4H-SiC Epilayers Using Homoepitaxial Web Growth
}

\author{
Philip G. Neudeck, David J. Spry ${ }^{1}$, Andrew J. Trunek ${ }^{1}$, J. Anthony Powell ${ }^{2}$, and \\ Glenn M. Beheim \\ NASA Glenn Research Center, \\ 21000 Brookpark Road, M.S. 77-1, Cleveland, OH 44135, U.S.A. \\ ${ }^{1}$ OAI, 21000 Brookpark Road, M.S. 77-1, Cleveland, OH 44135, U.S.A. \\ ${ }^{2}$ Sest, Inc., 21000 Brookpark Road, M.S. 77-1, Cleveland, OH 44135, U.S.A.
}

\begin{abstract}
This paper reports initial demonstration of a cantilevered homoepitaxial growth process that places screw dislocations at predetermined lateral positions in on-axis $4 \mathrm{H}-\mathrm{SiC}$ mesa epilayers. Thin cantilevers were grown extending toward the interior of hollow pre-growth mesa shapes etched into an on-axis $4 \mathrm{H}-\mathrm{SiC}$ wafer, eventually completely coalescing to form roofed cavities. Each completely coalesced cavity exhibited either: 1) a screw dislocation growth spiral located exactly where final cantilever coalescence occurred, or 2) no growth spiral. The fact that growth spirals are not observed at any other position except the central coalescence point suggests that substrate screw dislocations, initially surrounded by the hollow portion of the pre-growth mesa shape, are relocated to the final coalescence point of the webbed epilayer roof. Molten potassium hydroxide etch studies revealed that properly grown webbed cantilevers exhibited no etch pits, confirming the superior crystal quality of the cantilevers.
\end{abstract}

\section{INTRODUCTION AND BACKGROUND}

Axial screw dislocations are unpredictably distributed in high densities across all commercial 4H- and $6 \mathrm{H}-\mathrm{SiC}$ wafers [1]. In agreement with Frank's theory, screw dislocations with large Burgers vectors form hollow core "micropipes", while hollow cores are absent from screw dislocations with smaller Burger's vectors. Micropipe defects are well established to be unacceptable in $\mathrm{SiC}$ power devices, as junctions containing micropipe defects are unable to standoff high electric fields without excessive leakage current and microplasma formation [2]. Therefore, considerable efforts were undertaken with reasonable success to reduce micropipe densities in commercial $\mathrm{SiC}$ wafers to around 10 micropipes per square centimeter of wafer area [1]. However, much less attention has been paid to reducing the density of closed core screw dislocations, whose densities are more than 100 times more plentiful than micropipes in commercial $\mathrm{SiC}$ wafers. Closed core axial screw dislocations have also been shown to degrade the blocking properties of $\mathrm{SiC}$ high-field junctions, but to a much lesser degree than micropipes $[3,4]$. Therefore, commercialization of some $\mathrm{SiC}$ power devices containing closed core screw dislocation defects has been possible, as non-ideal device behavior has been largely dealt with by appropriate device design, yield screening, and blocking voltage de-rating [5]. Despite the successes in reducing micropipe densities and realizing modestly rated SiC power Schottky diodes on commercial $4 \mathrm{H}-\mathrm{SiC}$ wafers, there is nevertheless an increasing body of experimental evidence that non-micropipe structural crystal defects must be further reduced in order to realize even more beneficial $\mathrm{SiC}$ power devices $[3,4,6,7]$. 
Towards the goal of reducing structural defects in $\mathrm{SiC}$ epilayers, we recently reported a homoepitaxial $\mathrm{SiC}$ "web growth" technique that demonstrated lateral cantilever overgrowth of substrate micropipes and screw dislocation defects $[8,9]$. The cantilevered web growth process is briefly illustrated by the simplified cross-sections and optical micrographs of figure 1 . The process starts by dry reactive ion etching mesa patterns into a commercial on-axis (0001) $4 \mathrm{H}-\mathrm{SiC}$ substrate. The figure 1a cross-section (cutline shown across right arm) shows the bilayer steps that arise on the initial mesa surface due to the unintentional tilt angle of an on-axis polished substrate with respect to (0001) basal plane. Figure $1 \mathrm{~b}$ illustrates step-free mesa formation and evolution of thin cantilevers realized by carrying out epitaxial growth in pure stepflow conditions with no two-dimensional terrace nucleation and no screw dislocations to provide growth steps within the mesa $[9,10]$. Cantilevers form as growth adatoms, harvested by the large step-free surface, migrate to the mesa edges where the high bond density leads to incorporation into the crystal near the top of the mesa sidewall. The lateral propagation of the step-free cantilevered surface is significantly affected by pregrowth mesa shape and orientation, with the highest lateral expansion rates observed at the inside concave corners of mesas. When complete spanning of the interiors of $V$ 's (figure 1c) and other non-hollow mesa shapes (figure 1d) with concave corners by webbed cantilevers was accomplished, a step-free surface with significantly enlarged area over the pre-growth mesa was formed. Detailed analysis of webbed regions formed over a micropipe and closed-core screw dislocations show that caxis propagation of these defects is terminated by the laterally grown webbing $[8,9]$.

While the realization of enlarged stepfree $\mathrm{SiC}$ surfaces free of axial screw dislocations has some benefits (such as
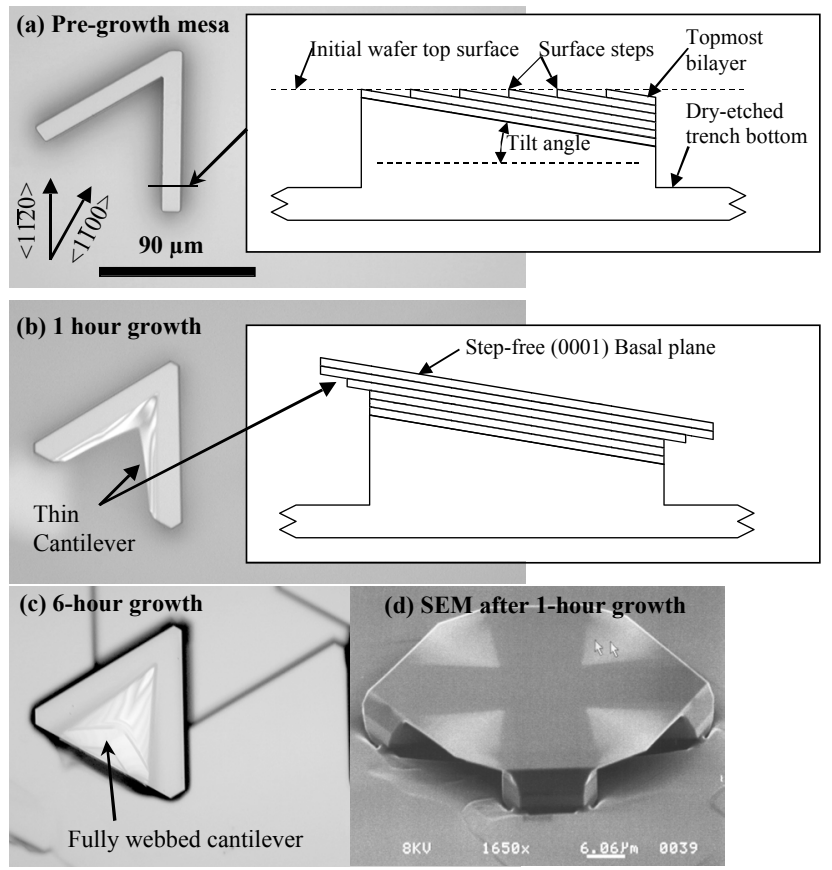

Figure 1. Optical micrographs, schematic crosssections, and SEM micrograph illustrating homoepitaxial web growth of thin $4 \mathrm{H}-\mathrm{SiC}$ cantilevers forming enlarged step-free (0001) basal plane surfaces on non-hollow mesa shapes $[8,9]$. heteroepitaxial growth discussed in [11]), it is important to note that such a surface does not support growth of thicker homoepitaxial SiC layers. In particular, because there are no screw dislocations present on the top of a step-free mesa surface to provide new growth steps, and hence a polytype replication template, all homoepitaxial growth in the c-axis direction ceases. When two-dimensional nucleation occurs on a basal plate terrace at growth temperatures below $2000{ }^{\circ} \mathrm{C}, 3 \mathrm{C}-\mathrm{SiC}$ (i.e., a heteroepitaxial layer) is produced [11,12]. Thus, our experiments in forming step-free mesas and webbed cantilevers are a confirmation that well-ordered homoepitaxial growth of hexagonal $\mathrm{SiC}$ polytypes up the c-axis cannot be carried out without screw dislocations providing new growth steps. However, where screw dislocations are present, the experiments reported in $[8-10,13]$ demonstrate that homoepitaxial growth of $4 \mathrm{H}-$ and $6 \mathrm{H}-\mathrm{SiC}$ 
can be carried out at substrate surface miscut angles as low as zero degrees (i.e., perfectly onaxis) or at temperatures as low as $1450{ }^{\circ} \mathrm{C}$.

This paper presents a modification of the previously reported web growth process that enables lateral dislocation overgrowth, yet also supports subsequent homoepitaxial growth of thicker $4 \mathrm{H}-\mathrm{SiC}$ layers on top of the webbed surface. In particular, this process should enable relocation, combination, and confinement of screw dislocations to predetermined lateral positions on top of a laterally webbed $\mathrm{SiC}$ mesa. In addition, this paper also reports the first defect preferential etching characterization of thin $4 \mathrm{H}-\mathrm{SiC}$ homoepitaxial cantilevers.

\section{EXPERIMENT}

The majority of processing and growth information for the sample presented in this work is given in [9], where it is identified as Sample \#5 in Table I. Pre-growth etching and subsequent epitaxial growth were carried out at $1600-1650{ }^{\circ} \mathrm{C}$ in a cold-wall horizontal growth system with uncoated graphite susceptor using $\mathrm{SiH}_{4}\left(2.7 \mathrm{~cm}^{3} / \mathrm{min}\right.$ during growth phase $)$ and $\mathrm{C}_{3} \mathrm{H}_{8}(0.3$ $\mathrm{cm}^{3} / \mathrm{min}$ ) in $\mathrm{H}_{2}$ (total flow $4400 \mathrm{~cm}^{3} / \mathrm{min}$ ) [9]. Growth rates for off-axis wafers are about 2 $\mu \mathrm{m} /$ hour for these conditions. However, in contrast to the open-ended "U" and "V" mesa shapes reported in [8,9], this paper focuses on results obtained on hollow enclosed-shape mesa patterns.

The upper right inset of figure 2 illustrates the hollow enclosed-shape pre-growth mesa pattern on the sample that was studied in the most detail. This raised mesa surface formed six hollow trench regions in the interior, each with equilateral triangle shape. Figure 2 shows a Nomarski optical micrograph (NOM) of the mesa following the 6-hour web growth process. The thin cantilevers have completely overgrown the hollow triangular trench regions, forming six roofed hollow cavities within the structure. Each of the six equilateral triangles is optically divided into three isosceles triangles by interference line features produced by the non-uniform thickness on the underside of the cantilevered film. These features are consistent with the "zippering" together of enlarging cantilevers from adjacent mesa arms of the structure [9]. Where the interference line features intersect each other around the center of each equilateral triangle represents the point where final coalescence of the cantilevered film occurred, completing the roof over each cavity. Some additional surface texture, such as the texture faintly visible in the geometric center of the hexagon, is observed on both webbed and support mesa portions of the structure. This texturing is optically consistent with surface texture previously associated with on-axis growth emanating from

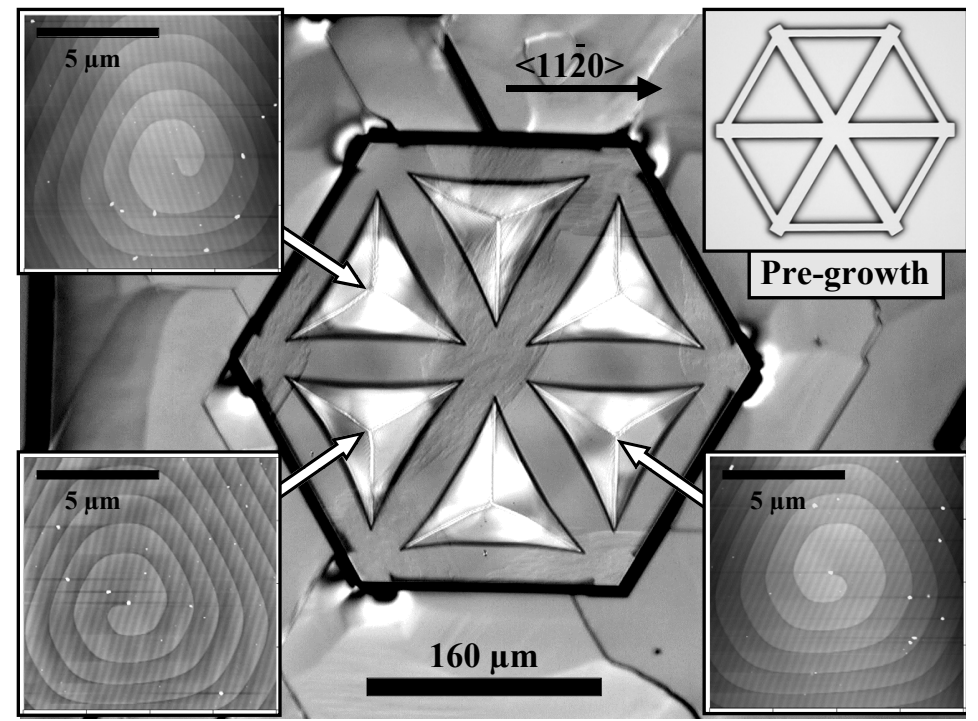

Figure 2. Optical micrograph of spoked hexagonal mesa following web growth that completely overgrew all six enclosed triangular trench regions. The AFM insets show the three growth spirals that formed exactly where roof closures occurred. 
screw dislocation hillocks, commonly observed to represent a downhill bunching of steps 10 to $100 \mu \mathrm{m}$ away from the peak of a hexagonal growth hillock [10].

The surface of the webbed mesa of figure 2 was extensively studied by atomic force microscopy (AFM). In contrast to step-free surfaces observed for non-enclosed mesa shapes (such as shown in figure 1) [8,9], an abundance of growth steps were observed across the entire figure 2 mesa. Three similar growth spirals, each shown in insets of figure 2, were found to be the sources for the steps observed on the mesa surface. All three growth spiral centers exactly corresponded to the lateral point of final cantilever coalescence near the center of a respective triangular hollow region, as illustrated in figure 2. The height of each AFM step in figure 2 is 1 $\mathrm{nm}$, corresponding to the c-axis stacking repeat distance of $4 \mathrm{H}-\mathrm{SiC}$. The growth step spiral indicates that an elementary screw dislocation formed in the film roof at these three respective points of film coalescence. No other growth spirals were observed elsewhere on the mesa surface, even over the pre-growth mesa and the other three cantilever coalescence points.

The sample was then subjected to a defect-preferential etch in molten potassium hydroxide $(\mathrm{KOH})$ at $500{ }^{\circ} \mathrm{C}$ for about 50 seconds. Figure 3 shows an optical micrograph of the figure 2 mesa following the $\mathrm{KOH}$ etch. The white arrows denote where three large hexagonal-shaped etch pits formed corresponding to the final web coalescence points where AFM detected growth spirals. The black arrows denote where four small hexagonal-shaped etch pits also formed in the mesa surface. These large pits and small pits are respectively consistent with previously reported $\mathrm{KOH}$ defect delineation etch studies of axial screw dislocations and other (i.e., non-screw) dislocations in $\mathrm{SiC}$ epilayers [14]. One of the smaller etch pits (P2 in figure 3) corresponds to a web coalescence point that did not exhibit a growth spiral. The other three smaller etch pits reside within pre-growth mesa regions.

Etch pits on the rest of the wafer, including numerous webbed cantilever films formed from nonenclosed "V", "U", and asterisk shaped mesas were also characterized $[8,9]$. Similar to figure 3 , small etch pits were often found on the pre-growth mesa

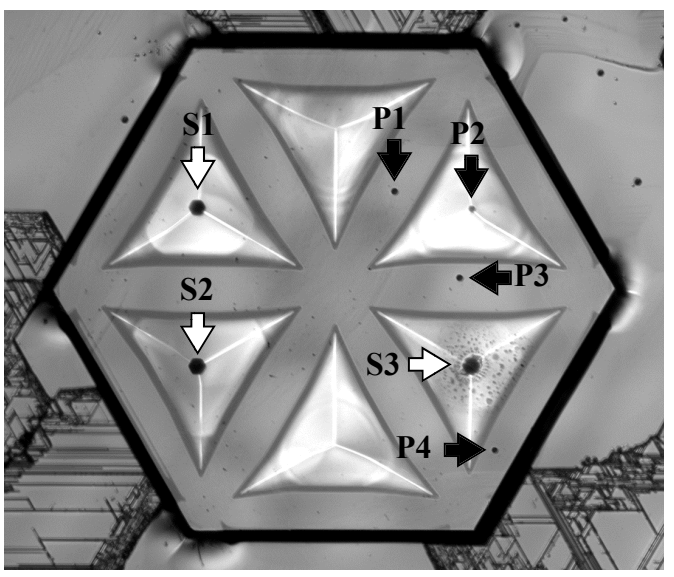

Figure 3. Optical micrograph of figure 2 mesa following a brief molten $\mathrm{KOH}$ etch. Three large hexagonal etch pits (white arrows) and four small hexagonal etch pits (black arrows) were observed on the surface. regions of fully webbed films, but were absent from all webbed regions except where (as with pit P2 in figure 3) webbed regions coalesced down to a point from more than two sides. No etch pits were observed for webbing that progressively coalesced outwardly on a non-enclosed mesa shape, such as what occurs for the V-shape and plus-shape mesas illustrated in figure 1. On mesas that did not form cantilevers, large etch pits (like S1 and S2 of figure 3) were found at the centers of the hexagonal growth hillocks produced by screw dislocations, further confirming that large etch pits and growth spirals corresponded to screw dislocations. The small etch pits, and corresponding absence of growth spirals, are consistent with other (i.e., non-screw) dislocations previously observed in SiC crystals by $\mathrm{KOH}$ etching [14]. Cantilevers entirely free of etch pits were achieved over areas sufficient for small prototype devices.

The wafer was then subjected to more aggressive molten $\mathrm{KOH}$ etching $\left(5\right.$ minutes at $500{ }^{\circ} \mathrm{C}$ followed by 10 minutes at $550{ }^{\circ} \mathrm{C}$ ) in an effort to remove the webbing and delineate substrate 
defects in the trench regions below webbed cantilevers. Figure 4 shows a scanning electron micrograph (SEM) of the figure 2 mesa following the additional etching. The webbing was completely removed from the triangular webs that contained screw dislocations (S1, S2, and S3 in figure 3). The smaller etch pits (such as P1 and P3 from figure 3) were greatly enlarged. The sidewalls of the support mesa experienced extensive etching, to the point where small etch pit $\mathrm{P} 4$ is no longer observable in figure 4 . The etching from small pit $\mathrm{P} 2$ resulted in a larger hole in the webbing. However, the aggressive $\mathrm{KOH}$ etch failed to remove, or reveal any additional features, in the two etch-pit-free triangular-shape web regions of the structure. Webbing was removed from all of the open-shaped structures (i.e., "U", "V", etc.) that permitted direct etching of the web from hanging outward edges of cantilevers (e.g., the lower edge of the web depicted in Fig 1c). The results are consistent with stepflow etching behavior, wherein the etch rate of the (0001) basal plane surface is insignificant compared to the etch rate of the sidewall surfaces and dislocations.

The white arrows of figure 4 show where deeper hexagonal etch pits formed in the substrate trench regions beneath webs that formed screw dislocations. The webbed mesa area $\left(\sim 6 \times 10^{-4}\right.$ $\mathrm{cm}^{2}$ ) was large enough that webbing should have overgrown at least one substrate screw dislocation

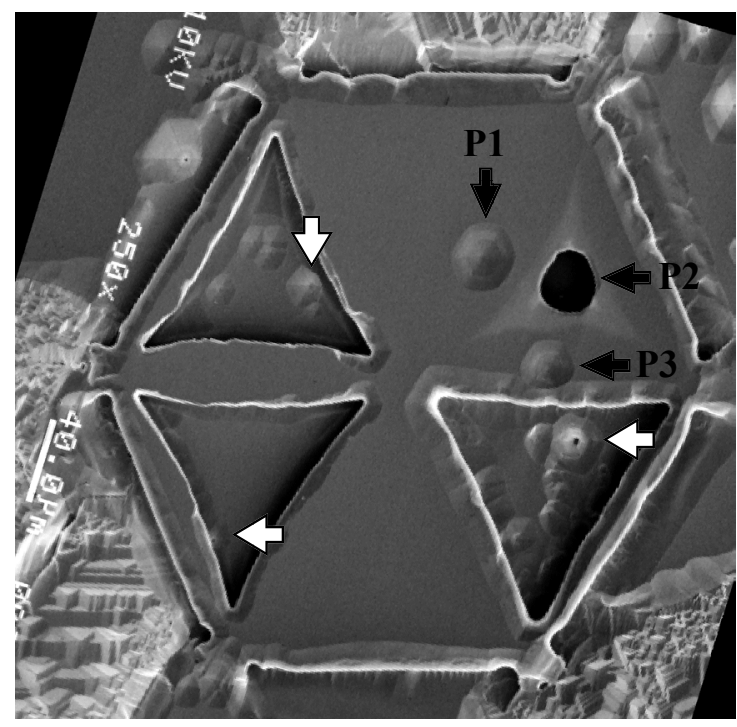

Figure 4. SEM of figure 2 mesa following additional molten $\mathrm{KOH}$ etching. Black arrows correspond to etch pit locations previously identified in figure 3 . in a trench region. However, it is not conclusive that the three white arrow etch pits correspond to screw dislocations. In particular, the final $\mathrm{KOH}$ etch greatly enlarged the pits due to non-screw dislocation defects (such as P1), preventing the clear distinction of etch pits corresponding to screw dislocations in the underlying trench regions.

\section{DISCUSSION AND SUMMARY}

Based upon the principle of Burgers vector conservation in a crystal [15], one could theoretically predict (consistent with experimental data) that at least one substrate screw dislocation resides beneath each of the enclosed-shape webs that form screw dislocations. The same conservation principle also leads us to speculate that when multiple screw dislocations are enclosed by a single hollow region of a pre-growth mesa shape, it may be possible to combine them into a single webbed film screw dislocation, wherein the Burgers vector of the film screw dislocation corresponds to the sum of the Burgers vectors of the enclosed substrate screw dislocations. Thus, we hypothesize that the use of a non-enclosed mesa shape, such as the "V" shape illustrated in figure 1., was essential for the previously reported realization of large atomically flat webs that overgrew screw dislocations without producing a spiral stepsource $[8,9]$. Further experiments are necessary to experimentally verify these theoretically predicted behaviors.

Nevertheless, the above experimental results clearly demonstrate that coalesced web growth from hollow enclosed mesa shapes can produce screw dislocations in predictable lateral 
locations. In particular, it has been shown that screw dislocations reside at the point of final lateral coalescence of webbed cantilevers. The point of final coalescence can be designed into the pre-growth mesa pattern using a basic understanding of the cantilever growth and faceting behavior of $\mathrm{SiC}$ as a function of crystallographic direction. Therefore, the process described in this paper enables SiC screw dislocations to be placed where they will not interfere with subsequently patterned devices. Following coalescence, the pre-placed screw dislocation can then provide steps for c-axis growth of on-axis homoepilayers on top of the mesa structure. However, it is important to recall that the pre-growth mesa itself (i.e., the raised pre-growth region) cannot contain a randomly located substrate screw dislocation in order for rapid webbing to occur. Therefore, it is important to minimize the pre-growth mesa area relative to substrate screw dislocation density while maximizing the designed area of the cantilever regions [9].

\section{ACKNOWLEDGEMENTS}

The authors would like to gratefully acknowledge the valuable assistance of Emye Benavage, Drago Androjna, Roger Meredith, Michelle Mrdenovich, Beth Osborn, Charles Blaha, John Heisler, Gary Hunter and Lawrence Matus at NASA Glenn Research Center, and Marek Skowronski and Tomas Kuhr at Caregie Mellon University, and Michael Dudley and William Vetter at State University of New York at Stony Brook. This work was funded by NASA Glenn

Research Center under the Computing Information and Communication Technology, Enabling Concepts and Technology, and Ultra Efficient Engine Technology programs.

\section{REFERENCES}

[1] A. R. Powell and L. B. Rowland, Proc. IEEE 90 (6), 942 (2002).

[2] P. G. Neudeck and J. A. Powell, IEEE Electron Device Lett. 15 (2), 63 (1994).

[3] P. G. Neudeck, W. Huang, and M. Dudley, Solid-State Electron. 42 (12), 2157 (1998).

[4] Q. Wahab, et al., Appl. Phys. Lett. 76 (19), 2725 (2000).

[5] R. Rupp, et al., in Silicon Carbide and Related Materials 1999, Materials Science Forum (Trans Tech Publications, Switzerland, 2000), Vol. 338-342, p. 1167.

[6] P. G. Neudeck, in Silicon Carbide and Related Materials 1999, Materials Science Forum (Trans Tech Publications, Switzerland, 2000), Vol. 338-342, p. 1161.

[7] H. Lendenmann, et al., in Silicon Carbide and Related Materials 2001, Materials Science Forum (Trans Tech Publications, Switzerland, 2002), Vol. 389-393, p. 1261.

[8] P. G. Neudeck, et al., in Silicon Carbide and Related Materials 2001, Materials Science Forum (Trans Tech Publications, Switzerland, 2002), Vol. 389-393, p. 251.

[9] P. G. Neudeck, et al., J. Appl. Phys. 92 (5), 2391 (2002).

[10] J. A. Powell, et al., Appl. Phys. Lett. 77 (10), 1449 (2000).

[11] P. G. Neudeck, et al., in Silicon Carbide and Related Materials 2001, Materials Science Forum (Trans Tech Publications, Switzerland, 2002), Vol. 389-393, p. 311.

[12] H. Matsunami, et al., in Amorphous and Crystalline Silicon Carbide, Springer Proceedings in Physics (Springer-Verlag, Berlin, Heidelberg, 1989), Vol. 34, p. 34.

[13] J. A. Powell, et al., Appl. Phys. Lett. 59 (3), 333 (1991).

[14] S. Ha, et al., J. Cryst. Growth 244 (3-4), 257 (2002).

[15] J. Friedel, Dislocations (Addison-Wesley, Reading MA, 1965), Chapter I, pp. 3-16. 